Giovanni Bruno

\title{
Vigilanza, alta vigilanza, sorveglianza: un campo (semantico) minato
}

Categoria di articoli: Microscopio

Citazione: Giovanni Bruno, Vigilanza, alta vigilanza, sorveglianza: un campo (semantico) minato, in: LeGes 31 (2020) 2 


\section{Indice}

1. Introduzione

2. «Vigilanza»

3. «Alta vigilanza»

3.1. Alta vigilanza in ambito amministrativo

3.2. Alta vigilanza in ambito parlamentare

4. «Sorveglianza»

5. Lo strano caso della sorveglianza degli assicuratori

6. Conclusione

\section{Introduzione}

[1] Se nel linguaggio comune i concetti di «vigilanza»e «sorveglianza» sono generalmente usati come sinonimi o risultano comunque intercambiabili nella maggior parte dei contesti, la situazione pare essere diversa in ambito giuridico e burocratico-amministrativo. Il presente contributo è un tentativo, necessariamente sommario in questa sede, di proporre una prima sistemazione terminologica in questo ambito caratterizzato da chiare competenze conferite dalla legge ma che fa un uso non sempre omogeneo della terminologia. In particolare si analizzeranno, mediante esempi tratti dalla legislazione federale, i termini «vigilanza», «alta vigilanza»e «sorveglianza».

[2] Proprio per l'approccio non esaustivo del contributo, non si entra nel merito di concetti quali «ispezione», "audit» o "verifica», che sembrerebbero rientrare alternativamente nelle categorie della vigilanza e della sorveglianza, e di «controlling», anglicismo usato per l'indigeno e usuale «controllo» (magari integrato con una specificazione del tipo «finanziario», "gestionale» ecc.) o per «supervisione». Il concetto stesso di «controllo», che a priori si presterebbe come iperonimo degli altri concetti qui trattati, in due casi dà invece addirittura il nome a organi operanti in questo senso in ambito finanziario e parlamentare: il «Controllo federale delle finanze» (CDF) e il «Controllo parlamentare sull'amministrazione» (CPA). La nostra analisi prescinde pure dalla nozione di controllo in senso lato quale contemperamento dell'azione dei singoli poteri garantita dalla loro separazione istituzionale, una sorta di controllo implicito iscritto nell'assetto costituzionale dello Stato di diritto e non a caso denominato anche sistema del «check and balance».

\section{2. «Vigilanza»}

[3] La vigilanza è esercitata da un'autorità istituzionale su un'altra autorità istituzionale o su privati. Il rapporto gerarchico tra le autorità in questione è variabile: la Confederazione può vigilare sull'Amministrazione federale e sui Cantoni, i Cantoni su enti di livello inferiore. D'altro canto, sia la Confederazione che i Cantoni possono vigilare sui privati. L'ente vigilante controlla essenzialmente che le leggi e altre disposizioni regolamentari siano rispettate e ha solitamente la facoltà di emanare istruzioni, regolamenti e altre normative ai fini della vigilanza. I corrispondenti tedesco e francese sono «Aufsicht» e «surveillance». Alcuni esempi emblematici:

Legge del 21 marzo 1997 sull'organizzazione del Governo e dell'Amministrazione (LOGA) (RS 172.010)

[4] Secondo l'articolo 8 capoverso 3, il Consiglio federale «vigila costantemente e sistematicamente sull'Amministrazione federale», mentre secondo il capoverso 4 «controlla, conformemente 
alle disposizioni particolari, le unità amministrative decentrate e gli organi esterni all'Amministrazione incaricati di compiti amministrativi della Confederazione». Mentre in entrambi i capoversi il tedesco si riferisce alla «vigilanza» («Aufsicht», «beaufsichtigen»), il francese parla di «surveillance» nel primo caso e di «contrôler» nel secondo (analogamente all'italiano).

Ordinanza del 25 novembre 1998 sull'organizzazione del Governo e dell'Amministrazione (OLOGA) (RS 172.010.1)

[5] La vigilanza da parte del Governo sull'Amministrazione, disciplinata nella sezione 5 comprendente gli articoli 24-27 (e intitolata, per l'appunto, «Vigilanza»), consiste sostanzialmente in un «controllo» («Kontrolle», «contrôle»), indicato quale «strumento della vigilanza» (art. 25 cpv. 1), come si evince dal testo degli articoli 25 e 26. Nell'articolo 27 è pure disciplinato un "controllo» (dei compiti della Confederazione), che nelle altre lingue è però detto «Überprüfung» $\mathrm{e}$, in modo concomitante, «contrôle»e «examen».

Legge federale del 22 giugno 2007 concernente l'Autorità federale di vigilanza sui mercati finanziari (Legge sulla vigilanza dei mercati finanziari, LFINMA) (RS 956.1)

[6] In questa legge, che istituisce un'autorità di vigilanza sui mercati finanziari ai sensi di nove leggi riassunte sotto l'etichetta di «leggi sui mercati finanziari» (art. 1: leggi sulle obbligazioni fondiarie, sul contratto d'assicurazione, sugli investimenti collettivi, sulla sorveglianza degli assicuratori e via dicendo), la Confederazione esercita la vigilanza sui privati.

[7] Facendo un breve excursus in campo fraseologico, notiamo che il titolo abbreviato potrebbe risultare fuorviante. Normalmente il verbo «vigilare» e il nome «vigilanza» richiedono la preposizione «su» (mentre «sorveglianza» vuole la preposizione «di»). La formulazione «vigilanza dei mercati finanziari» potrebbe far pensare che si tratti della vigilanza esercitata dai mercati finanziari, possibilità non scartabile a priori, e non di una vigilanza che un ente esercita sui mercati finanziari (come recita il titolo completo). È probabile che nel coniare il titolo abbreviato della legge si sia voluto evitare una cacofonia derivante dalla ripetizione della preposizione «su» già presente nella dicitura «legge su», che costituisce lo standard per i titoli abbreviati (pur non essendo tassativa). Su questa legge torneremo più avanti (n. 5).

Legge federale del 26 settembre 2014 concernente la vigilanza sull'assicurazione sociale contro le malattie (Legge sulla vigilanza sull'assicurazione malattie, LVAMal) (RS 832.12)

[8] Anche in questo caso la Confederazione esercita la vigilanza su enti privati.

\section{3. «Alta vigilanza»}

[9] L'alta vigilanza si colloca, come dice il termine stesso, a un livello superiore. Occorre tuttavia distinguere due ambiti, amministrativo e parlamentare, entro cui l'alta vigilanza si configura in modo differente e dà luogo a costellazioni diverse. Gli equivalenti tedesco e francese sono «Oberaufsicht»e «haute surveillance». 


\subsection{Alta vigilanza in ambito amministrativo}

[10] Un esempio concreto:

Codice civile (CC) (RS 210)

[11] Nell'ambito del registro fondiario la gestione degli uffici del registro fondiario sottostà alla vigilanza amministrativa dei Cantoni (art. 956 cpv. 1 CC) e all'alta vigilanza della Confederazione (art. 956 cpv. 2 CC). Concretamente, anche i cosiddetti delegatari privati, in particolare i fornitori di piattaforme alternative per la comunicazione elettronica, sottostanno agli stessi due tipi di vigilanza. Secondo l'articolo 6 capoverso 1 dell'ordinanza sul registro fondiario (RS 211.432.1), la Confederazione esercita l'alta vigilanza per il tramite dell'Ufficio federale per il diritto del registro fondiario e del diritto fondiario (UFRF).

[12] Secondo il documento esplicativo dell'Ufficio federale di giustizia intitolato «Delimitazione vigilanza e controlling dei Cantoni e alta vigilanza della Confederazione relative all'utilizzo del registro fondiario informatizzato da parte di delegatari privati», del 7 aprile 2014, la vigilanza da parte dei Cantoni è esercitata ad esempio sotto forma di controlli presso l'ufficio del registro fondiario o attraverso l'emanazione di disposizioni esecutive e di direttive su questioni amministrative e organizzative, mentre per l'alta vigilanza l'UFRF può emanare istruzioni circa l'esecuzione dell'ordinanza sul registro fondiario e delle relative disposizioni esecutive, procedere a ispezioni, controllare progetti e strategie dei Cantoni per la tenuta del registro fondiario, verificare l'idoneità e la conformità dei sistemi al diritto federale e fornire modelli per convenzioni sul cosiddetto accesso ampliato (pag. 4). Da notare che il «controlling» del titolo italiano corrisponde, rispettivamente, a «Controlling»e «contrôle».

[13] Un caso più complesso:

Legge federale del 29 settembre 2017 sui giochi in denaro (LGD) (RS 832.12)

[14] Secondo l'articolo 40 («Vigilanza», «Aufsicht», «Surveillance»), «l'autorità cantonale di vigilanza e d'esecuzione sorveglia il rispetto delle disposizioni legali concernenti i giochi di piccola estensione.» (cpv. 1). Notiamo che il verbo usato è «sorvegliare», che nelle versioni tedesca e francese corrisponde a «überwachen»e «contrôler».

[15] Vi è anche un'autorità federale di vigilanza, la Commissione federale delle case da gioco (CFCG), che «sorveglia» sia il rispetto delle disposizioni legali concernenti le case da gioco sia gli organi dirigenti e l'esercizio dei giochi delle case da gioco, il rispetto degli obblighi nel campo della prevenzione del riciclaggio di denaro e l'attuazione del piano di misure di sicurezza e del piano di misure sociali (art. 97 cpv. 1). È però chiaro che la CFCG in realtà «vigila»: infatti «riscuote ogni anno una tassa di vigilanza presso le case da gioco a copertura dei costi di vigilanza non coperti dagli emolumenti» (art. 99 cpv. 2).

[16] Dal canto suo, secondo l'articolo 104 capoverso 1 il segretariato della CFCG «esegue la vigilanza diretta sulle case da gioco». Il concetto di «vigilanza diretta» («unmittelbare Aufsicht», «surveillance directe») sembrerebbe significare la «sorveglianza» quale la descriviamo più sotto (n. 4). In merito si veda anche l'articolo 5 dell'ordinanza del 14 febbraio 2007 sugli esami genetici sull'essere umano (RS 810.122.1), secondo cui il capo di laboratorio esercita la «vigilanza diretta» sull'esecuzione di esami citogenetici e genetico-molecolari (pure qui «unmittelbare Aufsicht»e «surveillance directe»). 
[17] Ma esiste anche la cosiddetta «autorità intercantonale di vigilanza e d'esecuzione» che, in contrappunto all'operato dell'autorità cantonale di vigilanza e d'esecuzione di cui sopra, «sorveglia il rispetto delle disposizioni legali concernenti i giochi di grande estensione» (art. 105 cpv. 1 lett. a). D'altra parte, analogamente all'attività della CFCG descritta sopra, tale autorità «sorveglia» gli organi dirigenti e l'esercizio dei giochi da parte degli organizzatori di giochi di grande estensione, il rispetto degli obblighi nel campo della prevenzione del riciclaggio di denaro e l'attuazione del piano di misure di sicurezza e del piano di misure sociali (art. $107 \mathrm{cpv} .1$ lett. a).

[18] Infine, secondo l'articolo 138 capoverso 2, la Confederazione «esercita l'alta vigilanza» sull'esecuzione della legge.

[19] Nella legge sui giochi in denaro, possiamo dire, i rapporti tra vigilanti e vigilati ai diversi livelli sembrano muoversi entro un sistema di scatole cinesi.

[20] Un ultimo esempio di alta vigilanza in ambito amministrativo:

Ordinanza del 22 novembre 2006 concernente l'alta vigilanza sulla esecuzione e sul fallimento (RS 281.11)

[21] Secondo l'articolo 1, «l'Ufficio federale di giustizia esercita l'alta vigilanza sull'esecuzione e sul fallimento» ed è autorizzato a emanare istruzioni, circolari e raccomandazioni destinate alle autorità cantonali di vigilanza, agli uffici d'esecuzione e fallimenti nonché agli organi d'esecuzione esterni all'ufficio, per l'applicazione corretta e uniforme della legge, a redigere modelli per i moduli da utilizzare nelle procedure d'esecuzione e di fallimento e ad eseguire ispezioni delle autorità cantonali di vigilanza, degli uffici d'esecuzione e fallimenti nonché degli organi d'esecuzione esterni all'ufficio (lett. a-c).

[22] Si noti che l'autorità di alta vigilanza (federale) ha competenze direttive e ispettive anche nei confronti delle autorità di vigilanza (cantonali).

\subsection{Alta vigilanza in ambito parlamentare}

[23] Prendiamo anche qui le mosse da un esempio concreto. Nel rapporto del 28 giugno 2002 dal titolo «Alta vigilanza parlamentare sui tribunali federali» (FF 2002 6793) la Commissione della gestione del Consiglio degli Stati spiega che "per vigilanza va inteso il controllo esercitato da un'autorità o un organo vicini all'oggetto della vigilanza; si tratta di regola di una vigilanza interna esercitata in seno dello [recte: allo] stesso potere. Nel caso dei tribunali si tratta della vigilanza esercitata dall'autorità di grado superiore. L'alta vigilanza è invece una vigilanza esercitata da un potere su un altro potere» (pag. 6804, nota 5, corsivi originali).

[24] In questo ambito l'alta vigilanza ha caratteristiche diverse in quanto si attua tra poteri dello Stato e non all'interno dello stesso potere, nel qual caso si tratta di vigilanza. Nei rapporti che intercorrono tra i poteri dello Stato sembra quindi di poter individuare una dimensione verticale della vigilanza (tra organi appartenenti allo stesso potere) e una dimensione orizzontale dell'alta vigilanza (tra organi appartenenti a poteri diversi).

[25] Un esempio classico di alta vigilanza in quanto orizzontale è dato dall'alta vigilanza esercitata dal Parlamento sugli altri poteri dello Stato. Secondo l'articolo 26 capoverso 1 della legge sul Parlamento (RS 171.10), «l'Assemblea federale esercita l'alta vigilanza sulla gestione del Consiglio federale e dell'Amministrazione federale, dei tribunali della Confederazione, dell'autorità di vigi- 
lanza sul Ministero pubblico della Confederazione, del Ministero pubblico della Confederazione e di altri enti incaricati di compiti federali».

[26] Un altro esempio di alta vigilanza in quanto orizzontale è riconoscibile nella vigilanza esercitata, fino al 30 novembre 2019, dall'ormai sciolta Delegazione di vigilanza sulla NFTA (DVN). Secondo la legge del 4 ottobre 1991 sul transito alpino (RS 742.104, art. 20 cpv. 3-5, abrogati), la DVN ha esercitato l'alta vigilanza parlamentare sulla costruzione della Nuova ferrovia transalpina (NFTA). A rigore, dunque, si sarebbe dovuta chiamare «Delegazione di alta vigilanza sulla NFTA».

[27] È però opportuno evidenziare che la distinzione tra «vigilanza» in quanto verticale e «alta vigilanza» in quanto orizzontale è pertinente nei casi in cui è coinvolto il potere legislativo. Abbiamo infatti visto casi di alta vigilanza all'interno del potere esecutivo, ossia in senso verticale.

\section{4. «Sorveglianza»}

[28] La sorveglianza è un controllo diretto dell'attività dell'ente sorvegliato e interessa normalmente operazioni e processi concreti prescritti o autorizzati dalle normative legali. I correlativi tedesco e francese sono «Überwachung» e «surveillance».

[29] Un esempio eloquente:

Legge federale del 18 marzo 2016 sulla sorveglianza della corrispondenza postale e del traffico delle telecomunicazioni (LSCPT) (RS 780.1)

[30] In questo caso si tratta di un controllo diretto che implica l'impiego di apparecchi tecnici speciali e di programmi informatici speciali di sorveglianza del traffico delle telecomunicazioni secondo gli articoli 269bis e 269ter del Codice di procedura penale (RS 312.0). Questo tipo di sorveglianza, che mira ad accertare la commissione di illeciti o reati e che viola la sfera privata, va distinto dalla sorveglianza intesa al controllo dell'adempimento di quanto stabilito dalla legge. Il secondo tipo di sorveglianza è affidato alla Commissione delle poste, i cui compiti sono elencati all'articolo 22 capoverso 2 (lett. a-m) della legge del 17 dicembre 2010 sulle poste (RS 783.0):

b. verifica se le condizioni di lavoro abituali del settore sono rispettate e se vengono svolti negoziati per la conclusione di un contratto collettivo di lavoro (art. 4 cpv. 3 lett. b e c);

d. verifica il rispetto dell'obbligo d'informazione e dell'obbligo di fornire informazioni (art. 9 e 23);

e. sorveglia il rispetto del mandato legale di servizio universale (art. 13-17);

h. verifica il rispetto delle prescrizioni legali concernenti i prezzi del servizio universale (art. 92 cpv. 2, secondo periodo Cost., art. 16 cpv. 2 e 18 cpv. 3);

i. verifica il rispetto del divieto di sovvenzionamento trasversale (art. 19).

[31] Al verbo «verificare» delle lettere $b, \mathrm{~d}, \mathrm{~h}$ e i corrispondono da un lato «überwachen» (lett. b, d, h, i) e dall'altro «vérifier» (lett. b, d, h) e «surveiller» (lett. i). Al verbo «sorvegliare» della lettera e corrispondono «beaufsichtigen» e «surveiller». Al di là dello scarto terminologico determinato da «surveiller» alla lettera i (rispetto a «vérifier»), risulta alquanto arduo cogliere la 
differenza tra i compiti descritti alle lettere d ed e: in entrambi i casi si controlla il rispetto di norme specifiche, ma ciascuna versione linguistica usa due verbi diversi («verificare-sorvegliare», «überwachen-beaufsichtigen», «vérifier-surveiller»).

[32] Troviamo un altro esempio di «sorveglianza» nella legge sul transito alpino citata sopra. Abbiamo visto che la DVN ha esercitato l'alta vigilanza sulla costruzione della NFTA. Ora, l'articolo 19 capoverso 1 stabilisce che «il Consiglio federale assicura la sorveglianza e il controllo del progetto NFTA». È probabile che anche quella esercitata dal Governo sia in realtà una «vigilanza». Il tedesco ha «Aufsicht» e il francese «surveillance».

\section{Lo strano caso della sorveglianza degli assicuratori}

[33] La mescolanza di termini rientranti nel campo semantico della vigilanza, dell'alta vigilanza e della sorveglianza, già osservata in alcuni esempi, si manifesta in modo lampante in una legge dal forte impatto economico e finanziario:

Legge federale del 17 dicembre 2004 sulla sorveglianza degli assicuratori (Legge sulla sorveglianza degli assicuratori, LSA) (RS 961.01)

[34] Il capitolo 5 (art. 46-63) disciplina la «Sorveglianza» esercitata dalla Confederazione sulle imprese di assicurazione e sugli intermediari assicurativi. L'articolo 46 definisce i compiti (cpv. 1 lett. a-g) dell'organo competente per tale sorveglianza, ossia l'Autorità federale di vigilanza sui mercati finanziari (FINMA):

a. vigila sul rispetto della legislazione in materia di assicurazione e di sorveglianza;

c. vigila sul rispetto del piano d'esercizio;

d. vigila affinché le imprese di assicurazione siano solvibili, costituiscano le riserve tecniche nel rispetto delle prescrizioni e amministrino e investano gli elementi patrimoniali in modo regolare;

e. vigila sull'esecuzione regolare della liquidazione dei sinistri disciplinata nelle disposizioni della legge del 19 dicembre 1958 sulla circolazione stradale concernenti l'assicurazione di responsabilità civile per i veicoli a motore.

[35] In quanto autorità di «vigilanza», dunque, la FINMA vigila. Anche se lo fa in virtù di una legge che disciplina la «sorveglianza». Nella versione tedesca, per l'attività della FINMA (Eidgenössische Finanzmarktaufsicht) non si usa il verbo «beaufsichtigen» ma «wachen über» (lett. a, c e d) e «überwachen» (lett. e), mentre il francese non ha «surveiller» ma «veiller à» (lett. a, c, d ed e).

[36] Ora, nella necessità di distinguere i concetti, là dove la legge si riferisce alla sorveglianza vera e propria («Überwachung») l'italiano, che ha già «sorveglianza» (per «Aufsicht»), ricorre al termine «vigilanza»: l'articolo 27 disciplina la «Vigilanza interna sugli affari» («Interne Überwachung der Geschäftstätigkeit», «Contrôle interne de l'activité»), mentre l'articolo 76 ha per oggetto la «Vigilanza sui rischi» («Überwachung der Risiken», «Surveillance des risques»). 
[37] Assistiamo quindi a un'inversione dei termini «vigilanza» e «sorveglianza» rispetto all'uso in ambito giuridico e burocratico-amministrativo. Per questa anomalia sembra però esserci un motivo storico.

[38] Con la revisione totale della legge, nel 2004, il titolo è stato modificato quanto alla denominazione dei soggetti sottoposti a sorveglianza. Il titolo precedente recitava infatti: «Legge federale sulla sorveglianza degli istituti di assicurazione privati (Legge sulla sorveglianza degli assicuratori, LSA)». Notiamo che il titolo abbreviato e l'abbreviazione sono stati mantenuti e che si parlava già di «sorveglianza» (e anche la versione tedesca aveva già "Aufsicht»). La stessa cosa si può dire della revisione totale del 1978. È interessante constatare che già la prima versione della legge, del 1885, parlava di «sorveglianza»: «Legge federale del 25 giugno 1885 sulla sorveglianza delle imprese private in materia di assicurazione (LSA)».

[39] Se per la revisione totale del 2004 possiamo affermare che il testo di partenza, base per le traduzioni italiana e tedesca, era in francese (dato verificabile nel sistema informatico del CPU, il Centro delle pubblicazioni ufficiali della Cancelleria federale), per la revisione del 1978 e per la prima versione del 1885 non ci è dato saperlo. Che in origine vi sia stato un calco su «surveillance» è tuttavia un'ipotesi che possiamo scartare (ad ogni modo, la parola «vigilanza» è comunque attestata fin dal Quattrocento; cfr. Grande dizionario della lingua italiana, a c. di S. Battaglia, s. v. «vigilanza»). Infatti, se ci riferiamo a quanto scriveva il Consiglio federale nel 1976 con riguardo alle origini della sorveglianza degli assicuratori, la situazione si chiarisce:

«La base costituzionale della sorveglianza delle società d'assicurazione private da parte della Confederazione, sussistente dal 1874, è costituita dall'articolo 34 capoverso 2 della Costituzione federale del tenore seguente:

Le operazioni delle imprese private nel ramo delle assicurazioni sono sottoposte alla sorveglianza e alla legislazione della Confederazione.»

(messaggio del Consiglio federale all'Assemblea federale del 5 maggio 1976 concernente una nuova legge federale sulla sorveglianza degli istituti assicuratori privati; FF 1976 II 859, in particolare 860).

[40] La versione tedesca mostra che fin dall'inizio si è parlato di «Aufsicht»:

«Die seit 1874 bestehende verfassungsmässige Grundlage für die Beaufsichtigung der privaten Versicherungsunternehmungen durch den Bund ruht in Artikel 34 Absatz 2 der Bundesverfassung (BV) und lautet wie folgt:

Der Geschäftsbetrieb .... von Privatunternehmungen im Gebiete des Versicherungswesens unterliegt der Aufsicht und Gesetzgebung des Bundes.»

(BBl 1976 II 873, in particolare 874).

[41] Constatiamo quindi che la pertinente base costituzionale aveva «sorveglianza» già agli albori della normativa in questione.

[42] La compresenza di «sorveglianza» e "vigilanza», invertiti rispetto alla norma, si spiega con il fatto che nel 2007 l'istituzione della FINMA e il suo mandato di vigilare anche sugli assicuratori, 
considerati alla stregua di istituti finanziari, hanno fatto sì che si producesse un conflitto terminologico risolto solo in parte. Come abbiamo visto, prima dell'istituzione della FINMA, quando il tedesco parlava di «Überwachung», l'italiano ha usato «vigilanza» perché «sorveglianza» era già usato per «Aufsicht». Sotto questo profilo il francese, che usa «surveillance» per entrambi i concetti e se necessario ripiega su «contrôle», non incontra le stesse difficoltà dell'italiano.

[43] Sarebbe ipotizzabile modificare la terminologia in occasione della prossima modifica (magari una revisione totale) della legge? In teoria sì, ma un simile passo sarebbe poco ragionevole tenuto conto della consuetudine ultracentenaria sancita nel titolo stesso della legge, che non sembra aver mai dato problemi, e del fatto che nelle due revisioni totali del 1978 e del 2004 il Legislatore vi ha evidentemente rinunciato.

\section{Conclusione}

[44] Probabilmente, più che fare chiarezza la presente esposizione della casistica relativa al campo semantico della vigilanza, dell'alta vigilanza e della sorveglianza ha suscitato perplessità. Per farsi un quadro preciso della situazione occorrerebbe procedere a un'analisi esaustiva della legislazione federale quanto alle costanti e alle variabili della vigilanza, dell'alta vigilanza e della sorveglianza. Il presente contributo va quindi inteso come un primo avvicinamento alla problematica.

[45] L'uso disomogeneo dei termini nasce da un lato da una certa incoerenza terminologica nell'assegnare un compito di vigilanza o alta vigilanza a un'autorità, mentre dall'altro è imputabile al fatto che la legislazione federale in francese non distingue tra «vigilanza» e «sorveglianza» così come descritti in questa sede, e quindi ha potuto influire sulla versione italiana che vi si è adeguata supinamente (anche se, come abbiamo visto, nel caso della legge sulla sorveglianza degli assicuratori la terminologia ha una tradizione consolidata). Più precisamente, la lingua francese li distingue sì concettualmente ma non terminologicamente. Pare comunque ragionevole stabilire le seguenti equivalenze:

$$
\begin{aligned}
& \text { vigilanza }=\text { Aufsicht }=\text { surveillance } \\
& \text { alta vigilanza }=\text { Oberaufsicht }=\text { haute surveillance } \\
& \text { sorveglianza }=\text { Überwachung }=\text { surveillance. }
\end{aligned}
$$

[46] Nel caso del francese, ma la verifica in realtà andrebbe sempre fatta, occorre quindi valutare di volta in volta se il termine «surveillance» sia inteso come «vigilanza» o «sorveglianza», mentre per «haute surveillance» non ci sono dubbi: è l'«alta vigilanza».

Giovanni Bruno, Cancelleria federale, Servizi linguistici centrali, Divisione italiana, Bellinzona, e-mail: giovanni.bruno@bk.admin.ch. 